La formation professionnelle médicale doit répondre aux besoins de la population. Nous saluons tout soutien en vue d'améliorer I'efficacité et la qualité de la formation des médecins, à l'image du projet de l'Office fédéral de la santé publique (OFSP) présenté dans I'article ci-dessous.

Le catalogue des objectifs de formation de la Commission interfacultés médicale suisse (www.smifk.ch) a été élaboré selon des critères largement reconnus, les CanMEDS*. Les compétences requises ont ensuite été définies en fonction de ces objectifs. Les études de médecine ont donc dû être réformés et l'examen d'admission modifié en fonction du nouveau profil de compétence. La réussite de cet examen augmente de manière significative la probabilité d'achever ses études avec succès.

L'Institut suisse pour la formation médicale postgraduée et continue (ISFM) a également confié les objectifs généraux des programmes de formation postgraduée clinique à I'Institut d'ensei- gnement médical de l'Université de Berne (IML) pour que ce dernier les élabore sur la base des critères CanMEDS, c'est-à-dire en formulant les compétences à acquérir. Puis I'ISFM a envoyé ce projet en consultation auprès de toutes les sociétés de discipline médicale. L'étude initiée par l'OFSP va dans la même direction, voire éventuellement un peu plus loin: elle devrait servir à déterminer les compétences nécessaires pour soigner la population à I'avenir. Cette étude passe en revue les objectifs de l'ensemble de la formation professionnelle médicale. Nous recommandons une forte participation et attendons avec intérêt la lecture des résultats.

Dr Max Giger, président de I'ISFM

* Pour de plus amples détails au sujet de ces critères élaborés par le «Royal College of Physicians and Surgeons of Canada», veuillez consulter le site internet http://rcpsc.medical.org/canmeds/ CanMEDS2005/index.php.

\title{
Profils de compétences en médecine humaine et besoins sur le marché du travail
}

\author{
Quels sont les profils de compétences requis actuellement dans les différents domaines \\ de la médecine humaine? Quels seront-ils à l'avenir? Les formations de base et post- \\ grade existantes satisfont-elles aux exigences en la matière? Une étude, réalisée sur \\ mandat de I'OFSP, examinera ces questions. Voici un résumé des résultats provisoires \\ tirés de l'avant-projet.
}

\author{
Brigitte Menzi ${ }^{a}$, Volker Heyse ${ }^{b}$, \\ Arnulf Schircks, \\ Christoph Pfister ${ }^{d}$
}

a lic. rer. soc., collaboratrice scientifique, division Politique de la santé, OFSP

b Pr, directeur de la société $\mathrm{CeKom}^{\circledR} \mathrm{GmbH}$, Allemagne

c Dr phil., consultant en savoir-faire, Unterlunkhofen, Zurich

d Dr et MME (UniBE), responsable de la section Formation postgrade des professions médicales, OFSP

Correspondance:

Christoph Pfister, Dr et MME

(UniBE)

responsable de la section

Formation postgrade des pro-

fessions médicales, DFI, OFSP

Politique de la santé

Schwarzenburgstrasse 161

CH-3097 Liebefeld

Tél. 0313238731

Christoph.Pfister@bag.admin.ch
Compte tenu des capacités d'accueil limitées en études de médecine et dans le cadre des discussions en cours sur l'affectation du système de santé en professionnels adéquats, l'Office fédéral de la santé publique (OFSP) a identifié une lacune dans le programme qui pourrait être considéré comme idéal des formations de base et postgrade pour les différents domaines de la médecine humaine. La loi sur les professions médicales et le catalogue des objectifs de la Commission interfacultés médicale suisse définissent, à différents niveaux de détail, un certain nombre de compétences qui doivent être acquises au terme de chaque cycle de formation. Pour juger s'il est pertinent de restreindre le nombre de places d'études en sélectionnant certains profils d'étudiants (à l'aide d'un test d'aptitude aux études de médecine, par ex.), il faudrait savoir plus précisément de quels médecins le système de santé aura besoin à l'avenir et de quelles compétences non spécifiques ceux-ci devront disposer. On ne peut certes s'attendre à ce que les futurs spécialistes en médecine humaine disposent des mêmes compétences. Toutefois, ni en ce qui concerne les besoins du marché du travail, ni en ce qui concerne la transmission des compétences dans les formations de base et postgrade, on ne dispose de données empiriques exhaustives. L'OFSP a donc chargé la société $\mathrm{CeKom}^{\circledR}$, un centre de bilan de compétences
[1], de réaliser une étude pour répondre aux questions suivantes: Quels profils de compétences les différentes professions médicales nécessitent-elles? Les formations de base et postgrade existantes satisfont-elles aux exigences en la matière?

\section{Neuf tendances majeures dans le système de santé}

A la mi-décembre 2009, les deux premières phases du projet ont pu être terminées. L'étude principale portait sur le développement de méthodes. Se basant sur une vaste analyse bibliographique, elle expose les premières conclusions sur les exigences du marché du travail en termes de compétences et leur prise en compte dans les formations de base et postgrade des médecins. Neuf tendances majeures ont été identifiées. A l'avenir, elles caractériseront de manière déterminante la médecine et le profil de compétences en médecine humaine:

1. mutation démographique (vieillissement démographique, féminisation de la profession, vieillissement du corps médical [2]),

2. adaptation du système de santé aux réalités économiques en raison de la pression des coûts [3],

3. augmentation des soins ambulatoires [4],

4. application systématique de la gestion de la qualité, 
5 diversification de la profession de médecin (médecin en tant qu'entrepreneur, par ex.),

6. internationalisation de la santé,

7. nouveaux modèles de répartition des tâches,

8. gestion professionnelle des ressources humaines,

9. changement du discours sociétal sur la maladie et la santé.

Chacun de ces développements implique l'acquisition de compétences non spécifiques. Ainsi, par exemple, l'augmentation des soins ambulatoires entraînera non seulement de nouvelles procédures de travail et de conseils, mais aussi un besoin accru de coordination entre les secteurs ambulatoire et hospitalier. Par rapport au profil de compétences du médecin de famille, ces changements impliquent notamment que la mobilité ainsi que des capacités de planification, d'organisation, de coopération et de communication constitueront désormais des qualifications déterminantes.

Dans une deuxième étape, les compétences dégagées à partir de ces tendances ont été regroupées puis comparées avec celles du modèle canadien CanMED [5] et du cadre des compétences en leadership médical (Medical Leadership Competency Framework [6]). Cette analyse combinée a, dans un premier temps, permis d'identifier 46 compétences centrales non spécifiques. En parallèle, la société $\mathrm{CeKom}^{\circledR}$ a mené une série d'entretiens avec des experts qui ont permis de fournir un premier aperçu de l'évolution future des domaines d'intervention des spécialistes en médecine humaine. Pour ce faire, elle a tenu compte non seulement des domaines d'activité dans la prise en charge traditionnelle des patients (médecine curative, soins palliatifs, médecine des accidents, etc.), mais aussi des domaines qui ne nécessitent pas la présence du patient, tels que la recherche médicale, le conseil, l'administration ou l'industrie.

\section{Pas de temps pour les compétences fondamentales}

Concernant l'aspect de la formation, soit la question de savoir comment se déroulent aujourd'hui les formations de base et postgrade des spécialistes en médecine humaine et quelles compétences non spécifiques à la profession les médecins en formation acquièrent, $\mathrm{CeKom}^{\circledR}$ a notamment, dans les deux premières phases du projet, comparé les programmes de différentes universités suisses, puis mené des sondages écrits et oraux sur les formations de base et postgrade. Le bilan provisoire est le suivant: la promotion de compétences non spécifiques se fait essentiellement de manière implicite (au sens d'un «curriculum caché») dans la formation pratique et ne peut, de ce fait, ni être directement démontrée ni - a fortiori - faire l'objet d'un examen. Et, si les universités proposent déjà bon nombre de cours correspondants, les étudiants se rendent avant tout aux cours obligatoires, par manque de temps. En revanche, les établissements de formation, nombreux, connaissent une forte affluence.
Les approches isolées visant à renforcer les compétences non spécifiques sont toutefois parfaitement reconnaissables; les plus répandues sont certainement les stages. Une autre approche est la promotion de l'apprentissage en équipes de projet ainsi que de l'apprentissage autodirigé et auto-organisé. Par ailleurs, en séparant les cours obligatoires des cours optionnels et en axant des cours à part sur la promotion explicite de compétences non spécifiques, on améliorerait la situation. Les personnes interrogées ont avancé, comme obstacles principaux, une valorisation et une reconnaissance insuffisante de l'enseignement, trop peu de formation interprofessionnelle dans les cliniques et, dans l'ensemble, un coaching insuffisant des étudiants. Enfin, les personnes interrogées imputent ces carences aux professeurs et aux médecins-chefs; les uns négligent l'enseignement au profit de la recherche, les autres n'ont tout bonnement «pas de temps» pour la formation complète des médecins-assistants, formation qui serait également censée intégrer la transmission de compétences non spécifiques.

\section{On recherche experts}

L'élément central de la troisième phase du projet est constitué par l'élaboration concrète des profils visés, au cours de six ateliers d'experts organisés par l'OFSP et animés par $\mathrm{CeKom}^{\circledR}$. Si vous souhaitez vous exprimer sur ce thème, veuillez vous inscrire à l'adresse indiquée d'ici le plus rapidement possible. Pour vérifier les compétences non spécifiques, trois mesures de compétences doivent en outre être effectuées: une au début de l'étude, une autre à la fin (après l'année d'études à option) et la dernière au terme de la période de médecin-assistant. Le projet devrait s'achever d'ici à la fin avril 2011.

\section{Références}

1 Heyse V, Erpenbeck J. KompetenzManagement. Münster: Waxmann Verlag; 2007.

2 Kraft E. Statistique médicale 2008 de la FMH. Noueveau chifffres - nouveau contenu - nouvelles présentation. Bull Méd Suisses. 2009;90(12):455-61.

3 Hilbert J, Dahlbeck E, Enste P. Zukunftsmotor Gesundheitswirtschaft - ein Paradigmenwechsel von unten? In: Bogedan C, Müller-Schoell T, Ziegler A (Hrsg.). Demografischer Wandel als Chance. Erneuerung gesellschaftlicher Solidaritätsbeziehungen? Hamburg: VSA-Verlag; 2008. p. 62-81.

$4 \mathrm{H}+$ Die Spitäler der Schweiz. H+ (Hrsg.). Ambulante Behandlungen im Spital: Prügelknabe oder Dornröschen? URL: www.hplus.ch, aktualisiert 2009.

5 The CanMEDS 2005 Physician Competency Framework - Better Standards. Better Physicians. Better Care. 2005. Ottawa, The Royal college of Physicians and Surgeons of Canada.

6 Medical Leadership Competency Framework. Academy of Medical Royal Colleges.Institute for Innovation and Improvement (NHS) (Hrsg.). $2^{\text {nd }}$ edition. 2009. Warwick, Academy of Medical Royal Colleges. Institute for Innovation and Improvement (NHS). 